\title{
The Role of Apollon Gene Silencing on Viablity and Radiosensitivity of Cervical Cancer Hela Cells
}

\author{
Saeideh Milani ${ }^{1 *}$ Mojgan Bandehpour ${ }^{2}$, Zohreh Sharifi ${ }^{3}$, Bahram Kazemi ${ }^{2}$ \\ ${ }^{I}$ Shahid Beheshti University - Biotechnology Department Velenjak, Chamran highway, Tehran, Iran. ${ }^{2}$ Shahid \\ University - Biotechnology Department Tehram, Iran. ${ }^{3} 2$ Blood Transfusion Research Center - High Institute for \\ Research and Education in Transfusion Medicine, Tehran, Iran.
}

\begin{abstract}
Cervical cancer is the second common cause of cancer deaths in women worldwide. Radioresistancy of cancer is a principal cause of treatment impairing. Inhibitor of apoptosis proteins (IAPs) widely block apoptosis against apoptotic stimuli, including current chemo- and radiation therapies. Apollon, a membrane of IAP, can support cells against apoptosis and is over expressed in some treatment-resistant cancer cells.

The aim of this study was to evaluate the effects of apollon knockdown on induction of apoptosis and also its potential for enhancement of radiosensitvity on hela cells.

plasmid encoding shRNA which has been confirmed its effect against apollon, transfected into hela cells. Consequent effects on the level of P53, Bax and BAK analyzed by real time PCR. Apoptotic phenotype of transfected cells was monitored by Tunnel assay. Viability of hela cells after radiotherapy was analyzed by MTT assay.

shRNA1 effectively increased transcription of p53, Bax and BAK and induced apoptosis phenotype of treated hela cells. Radiosensitivity of transfected cell was increased after knock-down of apollon obviously.

Apollon knockdown induces apoptosis in hela cell . Also it can be as new molecular target for radio-sensitizing strategies in these cells. So, apollon can be a potentially considerable therapeutic object for cervical cancer.
\end{abstract}

Key words; Apollon, Apoptosis, Radiosensitivity, Cervical cancer

*Authors for correspondence: halle.mehr@gmail.com 


\section{INTRODUCTION}

The development of several cancers are tightly related to Human papillomavirus [HPVs] .This includes cervical cancer which is the second most common cancer in female population worldwide (Ma et al,2010). Apoptosis is an biological mechanism which results in programmed cell death (Sung et al,2007). Incontestable carcinogenesis and tumor progression are the results of apoptotic pathway malfeasance (Wei et al,2008).

Inappropriate apoptosis plays a significant role in the resistance of cancer cells to a wide range of contemporary anticancer therapies (Dong et al,2013). Chemo or radio resistance distinctly impairs the effectiveness of cancer therapy and includes anti-apoptotic signal transduction pathways that prevent cell death (Dai et al, 2009). One of the most apoptosis regulators which causing tumor cells resistant to apoptosis stimuli, like radiation and chemotherapeutic components, are IAPs [Inhibitors of Apoptosis] family (Hung et al,2008).

Apollon, also identified as Bruce or BIRC6, is the huge member of the IAP family which highly expressed in several types of cancer._Hu et al found that apollon knockdown in combination with chemotherapy may have therapeutic potential in the treatment of human colorectal cancer ( $\mathrm{Hu}$ et al,2015).

In another study, combinations of anticancer drugs with apollon-targeted therapy had efficient impact on treatment of esophageal cancer (Zhang et al,2014).

E6 viral gene is essential for the constant growth of cell lines derived from cervical cancers. E6 blocks cell apoptosis through directing the p53 tumor suppressor protein to the proteasome (Lehoux et al,2009).

Down regulation of $\mathrm{p53}$, leads to reduced apoptosis as well as higher tumor growth and development (Wong,2011).

A variety of treatments are recommended for cervical cancer include radiation therapy, surgery, and chemotherapy (Green et al,2007). The main target of radiation damage is DNA. After irradiation, p53 can detect DNA damage and be obviously increased. Thus, shortcoming in the p53 tumor suppressor gene have been associated with radio-resistance of cervical cancer (Fulda,2014).

In this study, the effect of apollon knockdown on promoting apoptosis in cervical cancer cells and subsequent increasing of sensitivity to radiotherapy in these cells was examined.

\section{MATERIAL AND METHODS}

\section{Cell lines}

Hela cell with the source of human cervical adenocarcinoma were obtained from Iran Blood Bank.The cells were grown in DMEM supplemented with 100 units $/ \mathrm{ml}$ penicillin (Sigma,USA)\& $\quad 100 \mu \mathrm{g} / \mathrm{ml}$ streptomycin (Sigma,USA) and $10 \%$ fetal calf serum (Gibco, Germany) at $37^{\circ} \mathrm{C}$ in $5 \%$ CO2humidified atmosphere.

\section{Transfection and real time PCR analysis}

In previous study, we constructed three shRNA insert (shRNA1,2 and 3) against apollon gene (Table 1) and transfected them into hela cell via electroporation method. In brief, at confluency of $70 \%, 100 \mu \mathrm{l}$ of hela cell suspension $\left(0.4 \times 10^{6}\right.$ cells/well) were transfected with $5 \mu \mathrm{g}$ of shRNAplasmids. For electroporation achievement, multiporator (Eppendorf, Germany) was used. Eelectrical field for transfer induction of shRNA into the cells was $200 \mathrm{~V}$ at $500 \mu \mathrm{s}$. Transfected cells were transferred into $1 \mathrm{~mL}$ of pre-warmed complete growth medium and incubated for 48 hours at $37{ }^{\circ} \mathrm{C}$ in a humidified incubator .

For doing real time PCR, total cellular RNA was extracted using Total RNA Purification Kit (Gena Bioscience, Germany) according to the manufacturer's instruction. cDNA synthesis was carried out, using Mmulv reverse transcriptase. Real time RT-PCR was performed with Accupower ${ }^{\mathrm{R}}$ 2X Greenstar QPCR Master Mix (Bio Neer,Korea). PCR reactions using specific primers under the following condition were designed: Initial 5 -min denaturation steps at $94^{\circ} \mathrm{C}$ and 45 cycles at $94^{\circ} \mathrm{C}$ for $5 \mathrm{~s}, 50^{\circ} \mathrm{C}$ for $8 \mathrm{~s}$ and 72 ${ }^{\circ} \mathrm{C}$ for $10 \mathrm{~s}$, using Rotor Gen 6000 (Corbett Research, Germany). Amplification of $\beta$-actin was carried out as internal control. Primer sequences are listed in Table 2.

Real time PCR results showed the mostly effective shRNA on apollon mRNA expression was shRNA1 (Milani et al,2016). 
Table 1- Sequence of shRNA against apollon mRNA

\begin{tabular}{ll}
\hline shRNA & sequence \\
\hline Apollon1 & CACCCTGCGCTCAACGCCATC \\
Apollon2 & CATGCTGGAATGTTGACGTTA \\
Apollon3 & TGGGAGATTGTTGCAAATG \\
\hline
\end{tabular}

\section{Expression levels of p53,Bax and Bak}

Relative mRNA expression of $\mathrm{p} 53$, bax and Bak genes between shRNA1- treated and pRNAin H1.2 NEO transfected cells [ mock control ], performed with specific primers ( Table 2) by the same protocol for apollon .

Table2- Primer sequences used for real-time PCR

\begin{tabular}{lr}
\hline Gene & Sequence \\
\hline Apollon & 5'-AGTGCAACGATGTGCCAT -3'/5'-GCTAACCAACAGAGAGTA-3' \\
P53 & 5'ACAATGGTTCACTGAAG3'/5'CTGTCCCAGAATGCAAG3' \\
Bax & 5'TTTCTGACGGCAACTAC3' '5'ATGTCCAGCCCATGATGG3' \\
Bak & 5'TTTACCGCCATCAGCAGG3' /5'TGCTGCAACATGGTCTGG3' \\
$\beta$-actin & 5'-GATGAGTATGCCTGCCGTGTG-3'/5'CAATCCAAATGCGGCATCT-3' \\
\hline
\end{tabular}

\section{Tunnel assay}

To evaluate cell death by apoptosis,tunel staining was performed using DeadEnd colorimetric kit (Promega) according to the instructions from the company. Cells $\left(5 \times 10^{3}\right)$ were transferred into polyl-lysine coated coverslips.After $24 \mathrm{~h}$,cells were transfected with mixture of liposome and shRNA1-plasmid at a 3:1 liposome:DNA ratio in 1 $\mathrm{ml}$ of serum-free media per transfection and incubated at $37 \mathrm{C}$ for one day.Then the coverslips were incubated in 4\%paraformaldehyde, permeabilised $(0.1 \%$ triton $\mathrm{X}-100$ in $0.1 \%$ sodium citrate) and then incubated with terminal deoxynucleotidyl transferase mediated dUTPbiotin for $1 \mathrm{~h}$ at $37 \mathrm{C}$. After rinsing with PBS, horseradish peroxidase (HRP) enzyme was added to coverslips at $37^{\circ} \mathrm{C}$ for $30 \mathrm{~min}$.After adding the peroxidase substrate, hydrogen peroxide, and the stable chromogen, diaminobenzidine (DAB) ,labeled DNA fragments were visualized and measured by fluorescence microscopy.

\section{Radiation sensitivity determination}

Cells were Grown $24 \mathrm{~h}$ in 8 -well chamber slides seeded with $10^{5}$ cells per well. Then,sh-RNA plasmid was transfected to hela cells using electroporation. mock control group was transfected with pRNAin H1.2neo vector alone. 24 $\mathrm{h}$ after transfection, Cells were irradiated with gamma-rays from a 60Co source under the sterile conditions at $37^{\circ} \mathrm{C}$. The irradiation dose was $4 \mathrm{~Gy}$ , a dose before revealed to be favorite for apoptosis induction in HeLa cells, and the dose rate was $2.92 \mathrm{~Gy} / \mathrm{min}$. The effects of irradiation on cell viability, was measured by MTT assay.

In brief, cells were covered with $20 \mu \mathrm{l}$ of 3-(4,5dimethylthiazol-2-yl)-2,5-diphenyltetrazolium bromide (MTT) $(5 \mathrm{mg} / \mathrm{ml})$ and incubated for an further $4 \mathrm{~h}$. For solubilizing the formazam crystals, $100 \mu \mathrm{l}$ of DMSO was added. The absorbance measurement was performed at 490 $\mathrm{nm}$ wavelength and reference wavelength of 620 $\mathrm{nm}$ in a microplate reader (Tecan, Sweden).

\section{Statistical analysis}

All statistical analyses were performed by using SPSS16. Each experiment was carried out in triplicate for all data $(n=3)$. Differences between control and shRNA-transfected cells in terms of growth and viability of the cells were analyzed using one-way analysis of variance (ANOVA) and independent samples t-test. Results were considered statistically significant at $\mathrm{P}<0.05$. Also, an average expression value ( $E$ value) indicating gene regulation was calculated using REST 
software, and $95 \%$ confidence intervals was used for expression ratios.

\section{RESULTS}

We used pRNAin H1.2 Neo vector system which is designed to facilitate the cloning of the doublestrand DNA oligonucleotide which is transcribed into shRNA by RNA polymerase III promoter once expressed in cells.

\section{Quantitative analysis of p53, bax and bak}

In previous study, we primarily have seen that expression of shRNAs for apollon markedly reduces the mRNA levels of this gene by mean factor of 0.02 in transfected hela Cell (Milani et al,2016). In this study, we evaluated the effects of apollon gene silencing on expression level of bax,bak and P53 using real time PCR. The analysis of RT-PCR using REST software showed that the expression of bax,bak and P53 mRNA in hela cells transfected with the apollon shRNA1, was increased significantly by mean factor of 18.5,52.3 and 35 ( $1.164,7.67$ and 1.166 fold) respectively compared to the mock control group.(Figure1).

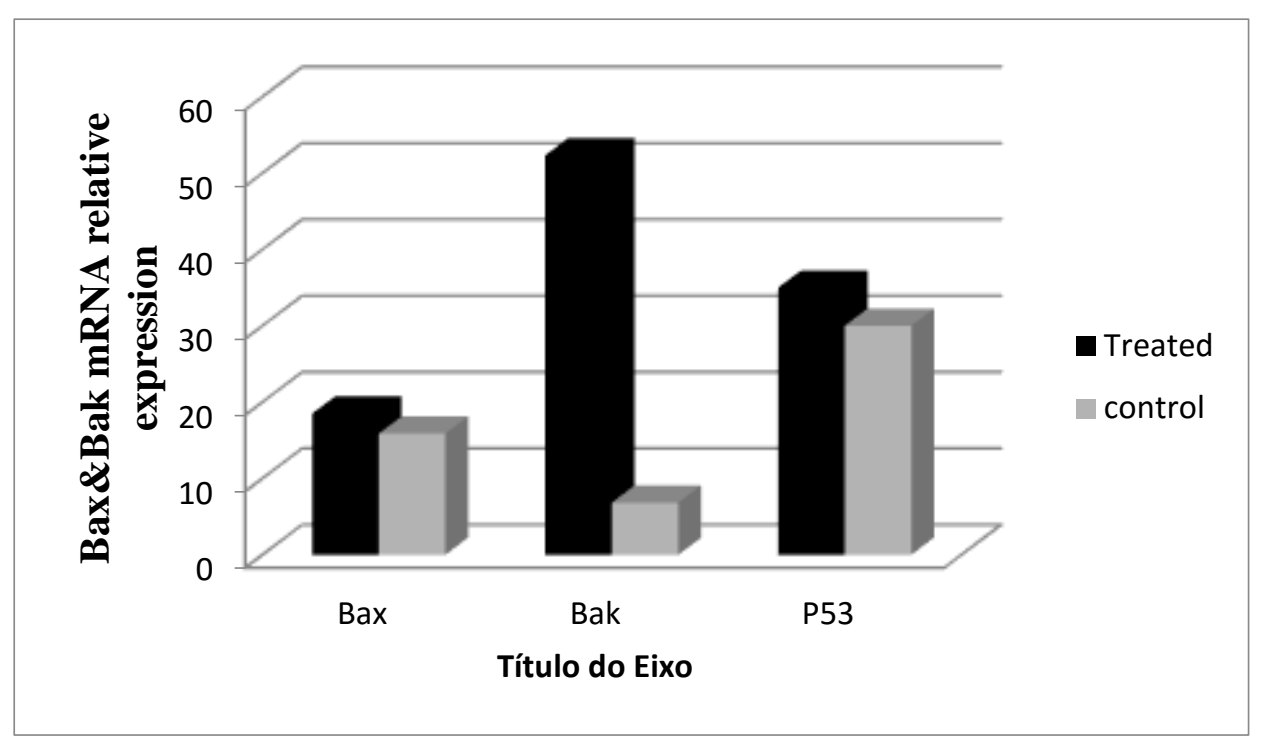

Figure1 - Up regulation of P53,Bax and Bak after knock down of apollon was shown after transfection the hela cell with shRNA1-plasmid. The mRNA expression of above genes was normalized with $\beta$-actin. An average expression value ( $E$ value) indicating gene regulation was calculated using REST software, and $95 \%$ confidence intervals were used for expression ratios.

\section{Fluorescence microscopy and TUNEL assays}

To affirm the apoptosis induction after knock down of apollon gene, Tunel assay was carried out. Nuclear DNA fragmentation, a significant biochemical indicator of apoptosis was calculated. The fragmented DNA of apoptotic cells is endlabeled with TUNEL (TdT-mediated dUTP NickEnd Labeling) assay. Biotinylated nucleotide is entered at the $3^{\prime}-\mathrm{OH}$ DNA ends with Terminal Deoxynucleotidyl Transferase (TdT). Horseradish- peroxidase-labeled streptavidin (Streptavidin HRP) is then linked to these biotinylated nucleotides, and then manifested using the peroxidase substrate, $\mathrm{H} 2 \mathrm{O} 2$, and the stable chromogen, diaminobenzidine (DAB). So apoptotic nuclei were stained dark brown. Tunel staining revealed that, apoptotic nuclei and apoptotic positive cells were prevalent in transfected samples (Figure 2). 


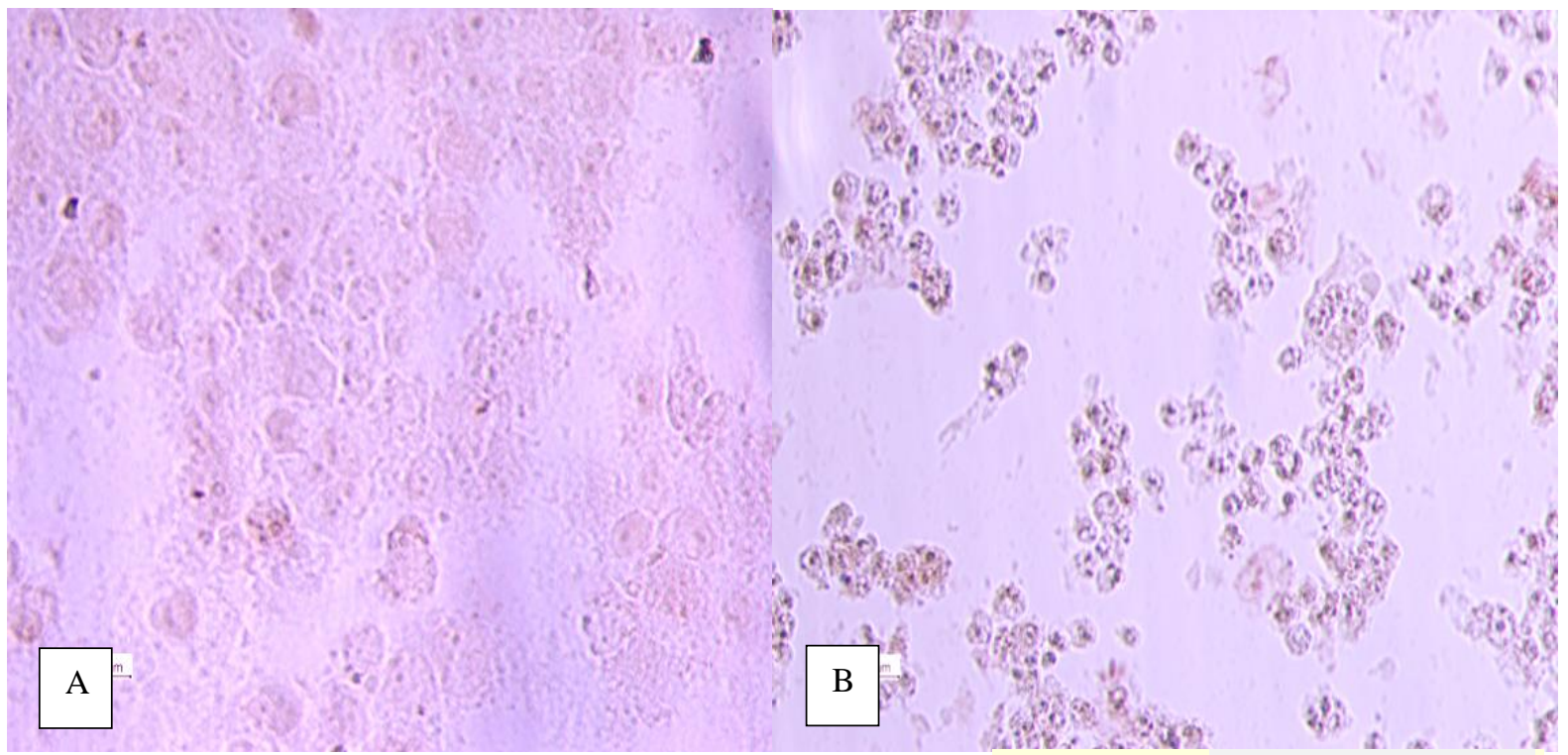

Figure2 - Tunnel staining for detection of apoptosis and apoptotic nuclei. apoptotic cells was stained dark brown. A) Control cells .B) shRNA1 transfected cells

\section{Sensitivity to radiotherapy}

More importantly, we examined the effect of combined treatment of IR with knock down of apollon in hela cancer cell lines for the first time.In the MTT assay, the cell growth inhibition of cervical hela cancer cells treated with both IR and shRNA against apollon was considerably greater than untransfected and shRNA treated alone (Figure3).

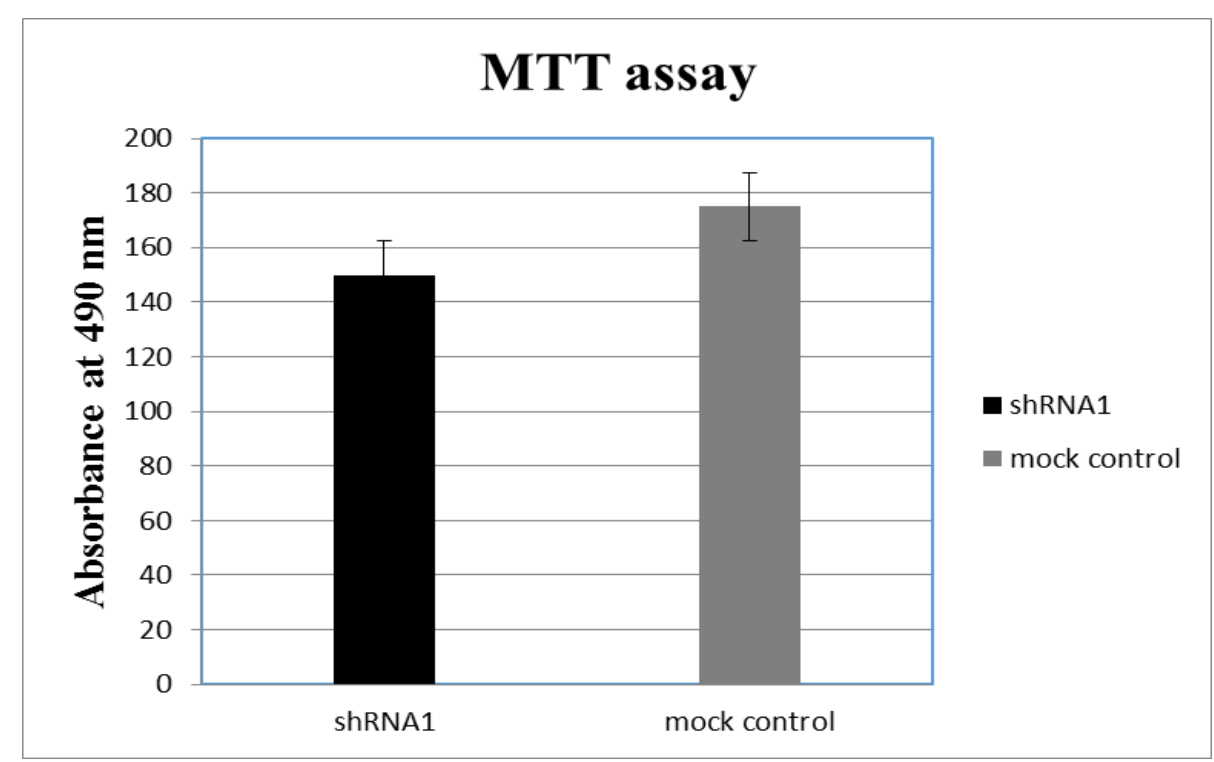

Figure3 - Apollon down-regulation on viability of hela cell after radiotherapy. cell viability was measured using MTT assays. Each bar represents the mean value \pm standard deviation (SD) of triplicate. $P<0.05$ was compared to the control cell group. 


\section{DISSCUSION}

HPVs plays a siginificant role in the development of servical cancers which is the second most common cancer in females worldwide(Hung et al,2008). There are no treatments that focus on cells containing HPV DNA, as a result, HPVinduced tumors are basically treated by surgery along with radiotherapy or nonspecific chemotherapy( Green et al 2007).

The resistance of tumor cells to apoptosis may trigger drastic clinical challenges. High-risk features at diagnosis and a poor response to various treatments, such as chemotherapy and radiotherapy can be associated with such situations (Wang et al,2009). Radiotherapy as a treatment option has been suggested for patients with locally advanced cervical carcinoma leads to progressionfree survival rates of 70\% (Wang et al,2009; Saxena et al,2005 ). A wide range of stimuli can activate the process of apoptosis. For example, radiation has been shown to be one such initiator. ( Saxena et al,2005 )

IAPs crucially contributes to the regulation of apoptosis. Several antiapoptotic proteins are expressed in different tumors, and their expression levels may be varied depend on the negative features at diagnosis and/or a poor response to treatment (Sung et al,2007).

Apollon, as a member of IAP, has a significant role in apoptosis inhibition and is up regulated in specific tumors, so its protein may be a favorable molecular target for treatment of cancers (Chu,2008). The goals for RNAi views for cancer therapy are ultimately to stop the expression of a cell cycle gene and/or an anti-apoptotic gene in the cancer cells leads to ending tumor growth and destroying the cancer cells (wang et al,2008).

Effective RNAi can be accomplished not only by transfection of siRNAs, but also by presentation of shRNA (short hairpin RNA) expressing plasmid into human Cells ( McIntyre et al,2006).

In previous study, we primarily have seen that expression of shRNAs for Apollon markedly reduces the mRNA levels of this gene in transfected hela Cell (Milani et al,2016). In this study, we evaluated the effects of Apollon gene silencing in this cell line.

Results of tunel assay showed that the loss of Apollon increased the apoptotic phenotype in transfected Cell.Using real time PCR revealed that the decrease in the levels of apollon caused an increase in the levels of p53 and proapoptotic genes bax and bak in transfected cells.

Expression of E6 protein from hela cells containing human papilloma virus leads to p53 degradation (25) (Niiforovi et al, 2004). our observation may be explained by the fact that Apollon knock down impairs the growth of cells markedly. This finding is parallel by upregulation of $\mathrm{p} 53$ and activation of the mitochondrial apoptotic pathway including Bax and bak upregulation.More importantly, we examined the effect of combined treatment of IR with knock down of apollon in hela cancer cell lines for the first time.

In the MTT assay, the cell growth inhibition of cervical HeLa cancer cells treated with both IR and shRNA against apollon was considerably greater than untransfected and shRNA treated alone.

In addition, High-level expression of the HPV16 E6 oncoprotein in tumors derived from transplanted human cervical carcinoma cells confers an aggressive radiation-resistant phenotype confirmed by former studies ( Jung et al,2015) .So, it is fair to assume that the observed reduction in the growth capability of tumor cells resulted from the knock down of apollon, upregulation of p53 and following induction of apoptosis.

The results of one study showed the important role of pollon in prostate cancer progression and treatment resistance, is and indicated for the first time that the BIRC6 gene and its product are potentially valuable targets for treatment of prostate cancers (Low et al,2013).

In another study, elevated apollon protein expression confirmed as predictive marker for chemoresistance of and poor prognostic factor for lung cancer patients (Dong et al,2013).

In clinical perspective, continuous treatment with effective IAP-inhibitors throughout the cycles of chemo/radiotherapy may reduce the risk of tumor local recurrence as well as metastasis. Therefore, a promising novel strategy to enhance the efficacy of present cancer treatments might be the combination of IAP-targeting molecular therapy and conventional chemo/ radiotherapy which ultimately improve the survival of cancer patients (Dai et al,2009).

Zhang et al found an inverse relationship between survivin- a member of IAP family- mRNA abundance and in vitro sensitivity to irradiation in pancreatic cancer cells (Zhang et al,2006). Wang 
showed that RNAi-mediated downregulation of XIAP [another member of IAP] expression can inhibit proliferation, induce apoptosis and moderate the radio-resistance of laryngeal carcinoma cells (Wang et al,2009).

Previous studies revolved around the impacts of apollon down regulation on sensitization of cancer cells to chemotherapy agents. Chu applied [ZD55siApollon] which is directed against apollon in hela, HT-1080 and MCF-7 cells. He found that ZD55-siApollon increased the antitumor effect of 5-fluorouracil, a chemotherapeutic agent (Chu,2008).Another study determined that apollon siRNA enhanced the chemosensitivity of HCC cells to the adriamycin and 5-fluorouracil (Chen et al,2011).

Given the fact that apoptosis is the main type of cell death induced by a variety of anticancer agents and IR. our study showed a potential and critical role of apollon as a factor of the radiosensitivity in cervical cancer cells. This study indicated that apollon knock down can induce apoptosis in hela cell line. Additionally, our results indicated a significant radiosensitivity in hela cell with down regulated expression of apollon up to now ( as recorded in literature).

\section{CONCLUSION}

Results of this study showed the effectiveness of combination treatment and subsequent enhancement of radio-sensitivity after apollon silencing in cervical cancer hela cell. Additional studies needed to validate apollon as a target of radiation sensitization in animal models of cervical cancer in a greater extent.

\section{ACKNOWLEDGEMENTS}

This project was supported by a Deputy in research of Shahid Beheshti University of Medical Sciences through grant No. 91-8414 and it was carried out in Cellular and Molecular Research Center and Biotechnology department of Shahid Beheshti University of Medical Sciences, Tehran, Iran

\section{REFERENCES}

Ma B, Xu Y, Hung CF, Wu TC. "HPV and Therapeutic Vaccines: Where are We in 2010?" Current Cancer Therapy Reviews. (2010): 6: 81-103.
Chen JF, Li YH, Chen YX, Xie KL. "Small interfering RNA targeting Apollon enhances the chemosensitivity of hepatocellular carcinoma cells in vitro." J South Med Univ (2011): 10:1701-1704.

Chu L, J. Gu. ""Oncolytic adenovirus-mediated shRNA against Apollon inhibits tumor cell growth and enhances antitumor effect of 5- fluorouracil." ." Gene Ther (2008): 15(7): 484-94.

Dong X, Lin D, Low C, Vucic EA. "Elevated expression of BIRC6 protein in non-small-cell lung cancers is associated with cancer recurrence and chemoresistance." J Thorac Oncol (2013): 8(2):161170.

Gudkou, A V. "The role of p53 in determining sensitivity to radiotherapy." CANCER (2003): 3(2):117-129.

Hung CF, Ma B, Monie A, Tsen SW, Wu TC. "Therapeutic human papillomavirus vaccines: current clinical trials and future directions.. ." Expert Opin Biol Ther (2008): 8(4):421-39.

Green KL, Brown C, Roeder GE. " A cancer cellspecific inducer of apoptosis." Human Gene Therapy (2007): 18(6):547-61.

Sung KW, Choi J, Hwang YK, Lee SJ, Kim HJ. "Overexpression of Apollon, an Antiapoptotic Protein, Is Associated with Poor Prognosis in Childhood De novo Acute Myeloid Leukemia. ." Clin Cancer Res (2007): 13:5109-5114.

Lehoux M, D'Abramo CM, Archambault J. " Molecular Mechanisms of HPV-induced Carcinogenesis ." Public Health Genomics (2009): 12(5-6):268-80.

Low C. G, Luk I. S. U, Lin D, Fazli L, Yang K, Xu Y, Wang Y. " BIRC6 Protein, an Inhibitor of Apoptosis: Role in Survival of Human Prostate Cancer Cells." PLoS ONE (2013): 8(2): e55837. .

Milani S, Bandehpour M, Sharifi Z, Kazemi B. " Suppressive effect of constructed shRNAs against apollon on apoptosis \& growth inhibition of cancer cell line. ." IBJ. 2016 Jan 10.Pii-IBJ-A-10-1599-1.

Wong RS. "Apoptosis in cancer: from pathogenesis to treatment." J Exp Clin Cancer Res (2011): 26:30-87.

Fulda S. " Targeting Inhibitor of Apoptosis Proteins for Cancer Therapy: A Double-Edge Sword?" JCO (2014): 32:3190-3191.

Saxena A, Yashar C, Taylor DD, Gercel-Taylor C. "Cellular response to chemotherapy and radiation in cervical cancer." Am J Obstet Gynecol (2005): 192(5):1399-403.

Hu T, Weng S, Tang W, Xue R. " Overexpression of BIRC6 Is a Predictor of Prognosis for Colorectal Cancer." PLoS ONE (2015): 10(5): e0125281.

Wang R, Li B, Wang X, Lin F, Gao P, Cheng SY, Zhang HZ. " Inhibiting XIAP expression by RNAi to inhibit proliferation and enhance radiosensitivity in laryngeal cancer cell line." Auris Nasus Larynx (2009): 36(3):332-339. 
Wei Y, Fan T, Yu M. "Inhibitor of apoptosis proteins and apoptosis." Acta Biochim Biophys Sin (2008): 40(4):278-88.

Dai Y, Lawrence TS, Xu L. "Overcoming cancer therapy resistance by targeting inhibitors of apoptosis proteins and nuclear factor-kappa B. ." Am J Transl Res (2009): 1(1): 1-1.

Yunbo Wei, Tingjun Fan, and Miaomiao Yu. "Inhibitor of apoptosis proteins and apoptosis." Acta Biochim Biophys Sin (2008): 278-88.

Zhang B, Liu JY, Pan JS, Han SP, Yin XX. "Combined treatment of ionizing radiation with genistein on cervical cancer HeLa cells." J Pharmacol Sci (2006): 102(1):129-135.

Zhang S, Tang W, Weng S, Liu X, Rao B, Gu J, Dong L. " Apollon modulates chemosensitivity in human esophageal squamous cell carcinoma ." Oncotarget (2014): 5(16): 7183-7197.
Mcintyre GJ, Fanning GC.Design and cloning strategies for constructing shRNA expression vectors.BMC Biotechnol 2006:6:1

Niiforovi A, Zari B , Daki A, Tima N. Flow Cytometry Evaluation of Hela S 3 Cell Death Induced by Gamma-radiation. Jugoslov Med Biohem( 2004): 23: $135-142$.

Jung HS, Rajasekaran N, Ju W, Shin YK. Human Papillomavirus: Current and Future RNAi Therapeutic Strategies for Cervical Cancer. J Clin Med. (2015) : 4(5): 1126-1155.

Received: October 17, 2015; Accepted: April 01, 2016. 